\title{
Эволюция примесной фотопроводимости в эпитаксиальных пленках CdHgTe при изменении температуры
} \author{
А.В. Галеева ${ }^{1}$, В.С. Варавин ${ }^{3}$, Н.Н. Михайлов ${ }^{3}$, С.А. Дворецкий ${ }^{3}$, \\ С.В. Морозов ${ }^{2}$, В.И. Гавриленко ${ }^{2}$ \\ ${ }^{2}$ Московский государственный университет им. М.В. Ломоносова, \\ 119991 Москва, Россия \\ ${ }^{2}$ Институт фризики микроструктур Российской академии наук, \\ 603950 Нижний Новгород, ГСП-105, Россия \\ ${ }^{3}$ Институт фризики полупроводников им. А.В. Ржанова \\ Сибирского отделения Российской академии наук, \\ 630090 Новосибирск, Россия \\ IE-mail: antikon@physics.msu.ru
}

(C) Т.А. Уаман Светикова ${ }^{1}$, А.В. Иконников ${ }^{1, \uparrow, ~ В . В . ~ Р у м я н ц е в ~}{ }^{2}$, Д.В. Козлов ${ }^{2}$, В.И. Черничкин ${ }^{1}$,

Поступила в Редакцию 24 апреля 2019 г.

В окончательной редакции 29 апреля 2019 г.

Принята к печати 29 апреля 2019 г.

В эпитаксиальных пленках $\mathrm{CdHgTe} \mathrm{исследованы} \mathrm{спектры} \mathrm{фотопроводимости} \mathrm{при} \mathrm{различных} \mathrm{темпера-}$ турах методом фурье-спектроскопии. В спектрах обнаружены особенности, связанные как с межзонным поглощением, там и с ионизацией примесно-дефектных состояний. Прослежена их эволюция с изменением температуры. Определены температуры „исчезновения“ примесных особенностей, что позволило, используя уравнение электронейтральности, оценить концентрацию акцепторов в исследуемых структурах.

Ключевые слова: фотопроводимость, примесь, $\mathrm{CdHgTe,} \mathrm{фурье-спектроскопия.}$

DOI: 10.21883/FTP.2019.09.48143.27

\section{1. Введение}

Твердые растворы $\mathrm{Cd}_{x} \mathrm{Hg}_{1-x}$ Те кадмий-ртуть-теллур (КРТ) широко применяется для создания приемников и приемных матриц среднего инфракрасного (ИК) диапазона. Одной из проблем при создании таких приемников высокой чувствительности на основе эпитаксиальных пленок КРТ является существенное влияние примесных и дефектных центров на времена межзонной рекомбинации. Эти центры исследуются уже свыше четырех десятилетий. Так, в ранних работах в слоях $\mathrm{Cd}_{x} \mathrm{Hg}_{1-x} \mathrm{Te}$ были обнаружены состояния вблизи края валентной зоны (акцепторные центры) [1]. Также в ряде работ [2-4] представлены результаты по наблюдению в спектрах фотолюминесценции и модуляционной спектроскопии переходов между глубокими примесно-дефектными центрами и мелкими акцепторными уровнями вблизи потолка валентной зоны. Однако следует отметить, что природа обнаруженных акцепторных центров осталась не выясненной.

Одним из методов изучения примесно-дефектных центров является фурье-спектроскопия фотопроводимости и фотолюминесценции, в том числе при различных температурах или в магнитных полях. Настоящая работа является продолжением серии исследований терагерцовой фотопроводимости (ФП) и фотолюминесценции (ФЛ) в объемных КРТ структурах. В работе [5] были исследованы спектры ФП при гелиевой температуре в эпитаксиальных пленках КРТ $p$-типа, с различной долей кадмия $(x=0.19-0.30)$ в твердом растворе. Во всех структурах в спектрах ФП были обнаружены субщелевые особенности, энергия которых практически не зависела от состава твердого раствора. Часть из этих особенностей связывалась с вакансиями ртути.

В работе [6] исследовались спектры ФЛ объемной КРТ пленки с долей кадмия в растворе 0.19 при температурах от 4.2 до $100 \mathrm{~K}$. Было показано, что положение длинноволновых линий ФЛ, связанных с захватом носителей на состояния в запрещенной зоне, не меняется и наблюдаемые линии гаснут с ростом температуры.

В настоящей работе исследованы спектры ФП в эпитаксиальных пленках КРТ $p$-типа с долей кадмия $x=0.22-0.23$ при различных температурах.

\section{2. Исследуемые образцы и методика эксперимента}

Исследуемые образцы $\mathrm{Cd}_{x} \mathrm{Hg}_{1-x}$ Te были выращены методом молекулярно-лучевой эпитаксии на подложке $\operatorname{GaAs}(013)$ в ИФП CO РАН [7,8]. На подложке выращивались буферные слои ZnTe и CdTe, затем выращивался активный слой структуры и, наконец, покровный слой CdTe. Параметры исследованных образцов представлены в таблице.

Выращенные образцы, по данным измерений эффекта Холла, обладали $n$-типом проводимости с концентрацией $\sim 2 \cdot 10^{14} \mathrm{~cm}^{-3}$ и подвижностью $\sim 50000 \mathrm{~cm}^{2} / \mathrm{B} \cdot \mathrm{c}$ при $T=4.2 \mathrm{~K}$. После роста структуры отжигались в атмосфере гелия при $T \propto 200^{\circ} \mathrm{C}$ в течение 24 ч $[9,10]$, 
Параметры исследуемых структур $\mathrm{Cd}_{x} \mathrm{Hg}_{1-x} \mathrm{Te}$

\begin{tabular}{c|c|c|c|c}
\hline Образец & $t$, мкм & $x$ & $E_{g}(T=0)$, мэВ & $x_{\mathrm{PC}}$ \\
\hline$A(120613)$ & 5.5 & 0.22 & 91 & 0.218 \\
$B(120621)$ & 6 & 0.23 & 105 & 0.226
\end{tabular}

Примечание. $t$ - толщина активного слоя, $x$ - номинальный состав структуры, $E_{g}$ и $x_{\mathrm{PC}}$ - ширина запрещенной зоны и состав твердого раствора, определенные из спектров фотопроводимости с учетом температурной зависимости ширины запрещенной зоны (см. формулу (5)).

что приводило к образованию значительного количества вакансий ртути, поскольку при отжиге атомы ртути покидают свои места из-за слабой связи $\mathrm{Hg}-\mathrm{Te}$. Вакансии ртути являются двухзарядными акцепторами, поэтому отжиг приводит к смене типа проводимости на дырочный. Концентрацию носителей при $T=4.2 \mathrm{~K}$ определить не удается, поскольку проводимость перестает носить металлический характер. Однако смена знака эффекта Холла в отожженных образцах при понижении температуры от комнатной к гелиевой отчетливо наблюдается, что говорит о смене типа проводимости.

Известно [10], что дополнительный отжиг в атмосфере ртути уже отожженных образцов приводит к ,залечиванию“ вакансий ртути и восстановлению концентрации электронов на уровне $2 \cdot 10^{14} \mathrm{~cm}^{-3}$. Поэтому мы будем полагать, что данная концентрация электронов вызвана полной ионизацией мелких доноров, энергия которых в КРТ не превышает 1 мэВ [11]. Это позволяет оценивать концентрацию доноров в исследуемых структурах как $2 \cdot 10^{14} \mathrm{~cm}^{-3}$.

Спектры фотопроводимости измерялись при низких температурах в характерном диапазоне 5-50 K с помощью фурье-спектрометра Bruker Vertex 70v. В качестве источника использовался глобар, в качестве светоделителя - Mylar Multilayer. Образцы размером $5 \times 4$ мм размещались в проточном криостате Oxford Instruments OptistatCF, который устанавливался в спектрометр так, чтобы образец находился в фокусе пучка излучения. Диаметр пучка составлял 0.5 мм. В криостате использовались окна из полипропилена и лавсана. Дополнительно перед образцом размещался холодный фильтр из черного полиэтилена. На образцы наносились полосковые индиевые контакты, через которые к образцу прикладывалось напряжение смещения около $500 \mathrm{MB}$. Сигнал фотопроводимости с образца усиливался с помощью усилителя Unipan 232B и подавался на аналогоцифровой преобразователь фурье-спектрометра. Спектры записывались с разрешением $4 \mathrm{~cm}^{-1}$.

\section{3. Метод расчета}

Для оценки температурной зависимости интенсивности спектральных линий, связываемых с вакансиями ртути, было рассчитано число акцепторных центров, участвующих в формировании сигнала фотопроводимости $\left(A^{0}\right.$ - и $A^{-1}$-центров) (см. $\left.[5,6]\right)$. Для этого решалось уравнение электронейтральности:

$$
\begin{gathered}
N_{v} \exp \left(\frac{E_{V}-F}{T}\right)+\frac{N_{d}}{1+2 \exp \left(\frac{F-E_{d}}{T}\right)}=N_{c} \exp \left(\frac{F-E_{c}}{T}\right) \\
+\frac{N_{A}\left(2+4 \exp \left(\frac{E_{A 1}-F}{T}\right)\right)}{1+4 \exp \left(\frac{E_{A 1}-F}{T}\right)+6 \exp \left(2 \frac{E_{A 2}-F}{T}\right)}
\end{gathered}
$$

где $T$ - температура в энергетических единицах; $F-$ химический потенциал; $N_{d}, N_{A}$ - концентрации доноров и акцепторов соответственно; $E_{d}, E_{A 1}, E_{A 2}-$ энергии соответственно донорного состояний и состояний $A^{-1}$ и $A^{0}$-центров;

$$
N_{V, C}=\frac{2\left(2 \pi m_{V, C} T\right)^{3 / 2}}{(2 \pi \hbar)^{3}}
$$

- эффективные плотности состояний в валентной зоне и зоне проводимости соответственно, $m_{V, C}$ - эффективные массы тяжелых дырок и электронов соответственно.

Правая часть уравнения - концентрация положительных зарядов, т.е. дырок и заряженных одновалентных доноров, левая часть - концентрация отрицательного заряда, т.е. концентрация электронов и плотность заряда, связанная с акцепторными центрами - концентрация ионизованных $A^{-1}$-центров и удвоенная концентрация двукратно ионизованных $A^{-2}$-центров. При расчете предполагалось, что полупроводник не вырожден, т.е. уровень Ферми отстоит от краев зон на энергию, намного превышающую температуру (в энергетических единицах), а распределение электронов и дырок в зонах подчиняется статистике Максвелла-Больцмана. Предполагалось, что полупроводник имеет проводимость $p$-типа, а концентрация компенсирующей донорной примеси составляет $N_{d}=2 \cdot 10^{14} \mathrm{~cm}^{-3}$. При этом энергия ионизации $A^{0}$ - и $A^{-1}$-центров составляла соответственно $\left(E_{A 2}-E_{V}\right)=11$ мэВ и $\left(E_{A 1}-E_{V}\right)=21$ мэВ (см. далее). Энергия ионизации доноров $\left(E_{c}-E_{d}\right)$ бралась равной 1 мэВ.

Из уравнения (1) находилось положение химического потенциала $F$, а затем рассчитывались концентрации $A^{-1}$-центров:

$$
N_{A^{-1}}=\frac{4 N_{A} \exp \left(\frac{E_{A 1}-F}{T}\right)}{1+4 \exp \left(\frac{E_{A 1}-F}{T}\right)+6 \exp \left(2 \frac{E_{A 2}-F}{T}\right)}
$$

и $A^{0}$-центров

$$
N_{A^{0}}=\frac{6 N_{A} \exp \left(2 \frac{E_{A 2}-F}{T}\right)}{1+4 \exp \left(\frac{E_{A 1}-F}{T}\right)+6 \exp \left(2 \frac{E_{A 2}-F}{T}\right)},
$$

участвующих в формировании сигнала фотопроводимости. 
Сигнал фототока пропорционален числу центров, с которых носители возбуждаются в зону, и времени жизни такого носителя в зоне: $j \propto N_{A^{0,-1}} \tau$. При этом время жизни $\tau \propto 1 / N_{\mathrm{ph}}$, где $N_{\mathrm{ph}}$ - число фононов. Мы не знаем, как именно происходит релаксация носителей и какие фононы принимают в этом участие, но будем считать, что релаксация дырок из валентной зоны происходит на очень мелкие акцепторные состояния. Таким образом, время жизни дырки в континууме - это время ее захвата на очень мелкий акцепторный уровень. Для такого процесса можно предположить, что энергия фонона много меньше температуры $\left(\hbar \omega_{\text {ph }} \ll T\right)$. В этом случае

$$
N_{\mathrm{ph}}=\frac{1}{e^{\frac{\hbar \omega_{\mathrm{ph}}}{T}}-1} \approx \frac{T}{\hbar \omega_{\mathrm{ph}}} .
$$

Тогда соотношение для фототока принимает вид $j \propto N_{A^{0,-1}} \tau \propto \frac{N_{A^{0,-1}}}{T}$. Отсюда, используя выражения (2) и (3), можно, задавая число акцепторов NA, определить температурные зависимости интенсивности спектральных линий, соответствующих $A^{-1}$ - и $A^{0}$-центрам.

\section{4. Результаты и обсуждение}

Результаты исследований спектров ФП в образце $A$ при различных температурах представлены на рис. 1 . Хорошо видна область межзонной фотопроводимости при $E>90$ мэВ и область примесной проводимости при меньших энергиях. Множественные провалы в области межзонной проводимости связаны с особенностями пропускания светоделителя в данном диапазоне. Красная

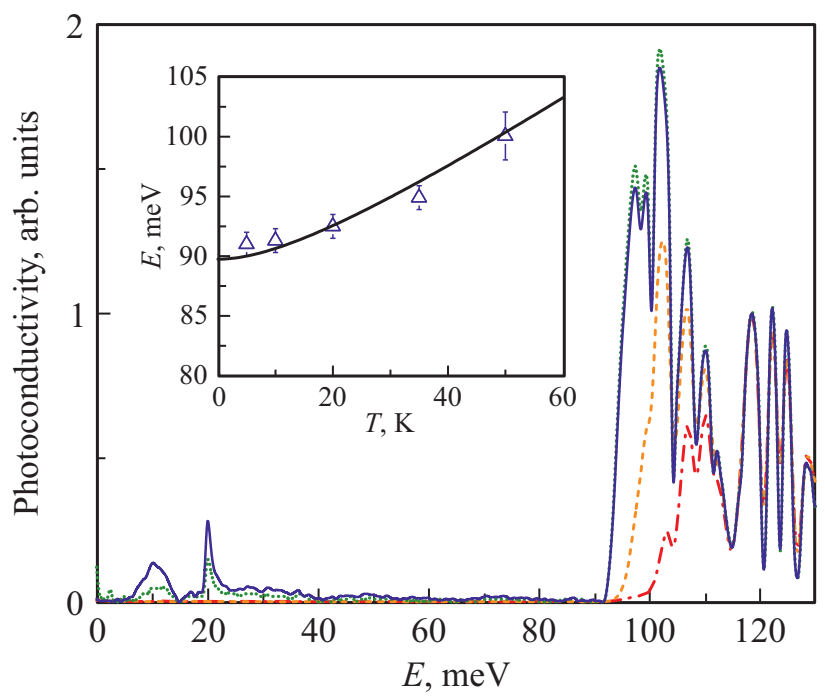

Pис. 1. Типичные спектры фотопроводимости в образце, измеренные в диапазоне температур 5-50 K: сплошная линия $5 \mathrm{~K}$, пунктирная - $6.5 \mathrm{~K}$, штриховая $-35 \mathrm{~K}$, штрихпунктирная - $50 \mathrm{~K}$. На вставке: температурная зависимость красной границы поглощения, линия - эмпирическая зависимость из работы [13]. граница межзонной фотопроводимости с ростом температуры сдвигается в сторону больших энергий, в то время как положение примесных линий не меняется, а меняется лишь их интенсивность.

Сдвиг красной границы поглощения в сторону больших энергий связан с увеличением ширины запрещенной зоны с ростом температуры в КРТ [12]. Температурная зависимость положения красной границы показана на вставке к рис. 1. Для аналитического описания данной зависимости мы использовали эмпирическую формулу из работы [13]:

$$
\begin{aligned}
E_{g}(x, T)= & -0.303(1-x)+1.606 x-0.132 x(1-x) \\
& +10^{-4} T^{2} \frac{6.3(1-x)-3.25 x-5.92 x(1-x)}{11(1-x)+78.7 x+T} .
\end{aligned}
$$

В качестве подгоночного параметра выступал состав $x$. Наилучшее согласие было достигнуто при составе $x=0.218$, что хорошо совпадает с номинальным составом (см. таблицу). Ширина запрещенной зоны для такого состава при $T=0 \mathrm{~K}$ равна $E_{g}^{A}=91$ мэВ. В образце $B$ состав раствора и ширина запрещенной зоны, определенные таким же методом, составили $x=0.226$ и $E_{g}^{B}=105$ мэВ соответственно.

Перейдем теперь к рассмотрению субщелевых особенностей спектров ФП. На рис. 2 и 3 показаны области примесной фотопроводимости в образцах $A$ и $B$ соответственно. Наблюдаемые особенности обозначены цифрами. Положения пиков в образцах $A$ и $B$ с хорошей точностью совпадают, однако интенсивности их отличаются: в общем случае в образце $A$ особенности 1 и 2 преобладают над особенностями 3,4 и 5 , а в образце $B$ наоборот. Вслед за авторами работы [5] мы придерживаемся следующей интерпретации данных особенностей. Пики 1 и 2 связаны с вакансией ртути двухзарядным акцептором. Пик 1 связан с отрывом первой дырки от нейтрального акцептора ( $A^{0}$-центр), пик 2 - с отрывом второй дырки от уже однократно ионизованного акцептора ( $A^{-1}$-центр).

Интерпретация особенности 3, которая представляет собой широкую спектральную полосу с максимумом на 30.2 мэВ, пока не однозначна. Предполагается, что она связана либо с какой-то другой примесью, либо с замещением вакансии ртути кислородом [5]. В данной работе мы не будем обсуждать ее эволюцию с температурой.

Пик 4 связывается с усилением поглощения из-за отражения излучения от подложки $\mathrm{GaAs}$ в области остаточных лучей [14], что приводит к возрастанию поглощения (и соответственно появлению особенности) в спектральной области вблизи 40 мэВ. Данная особенность не является „самостоятельной“, она лишь следствие усиления поглощения особенности 3 в области 40 мэВ.

Наконец, особенность 5, по всей видимости, имеет такую же природу, как и особенность 3. На это 
указывает их общее поведение с ростом температуры (рис. 2, 3), а также одинаковые изменения под действием других факторов (например, дополнительной подсветки видимым светом).

Помимо указанных особенностей, в спектрах примесной фотопроводимости наблюдаются особенности в виде провалов, связанных как с фононным поглощением на 15 и 18.7 мэВ [15], так и с поглощением в используемом черном полиэтилене на 29.6 и 40.7 мэВ. Отметим, что в

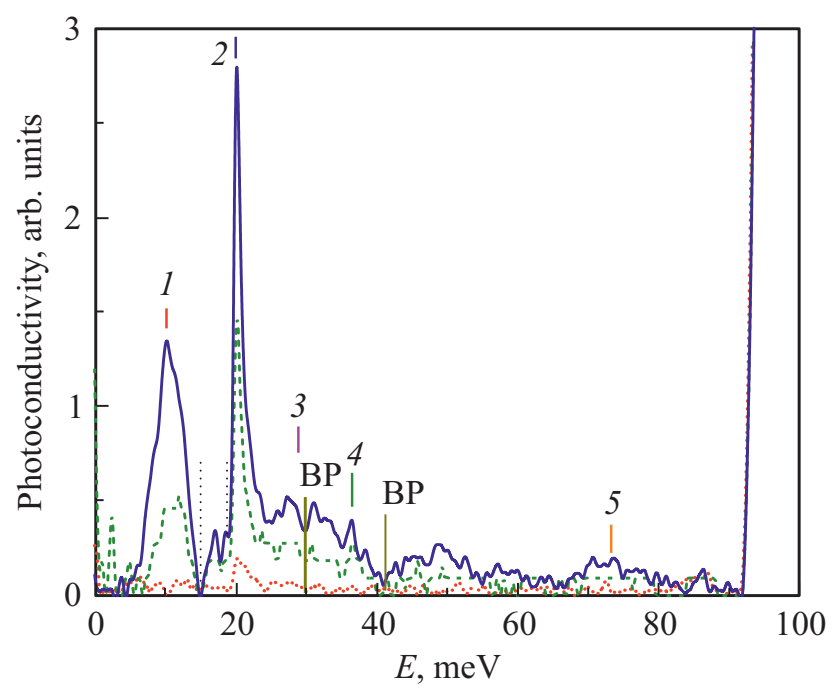

Рис. 2. Спектры примесной фотопроводимости в образце $A$ при различных температурах: сплошная линия $-5 \mathrm{~K}$, штриховая линия - $6.5 \mathrm{~K}$, пунктирная линия $-9 \mathrm{~K}$. Цифрами отмечены наблюдаемые особенности. Точечными вертикальными линиями отмечены области фононного поглощения. ВР области поглощения используемого черного полиэтилена.

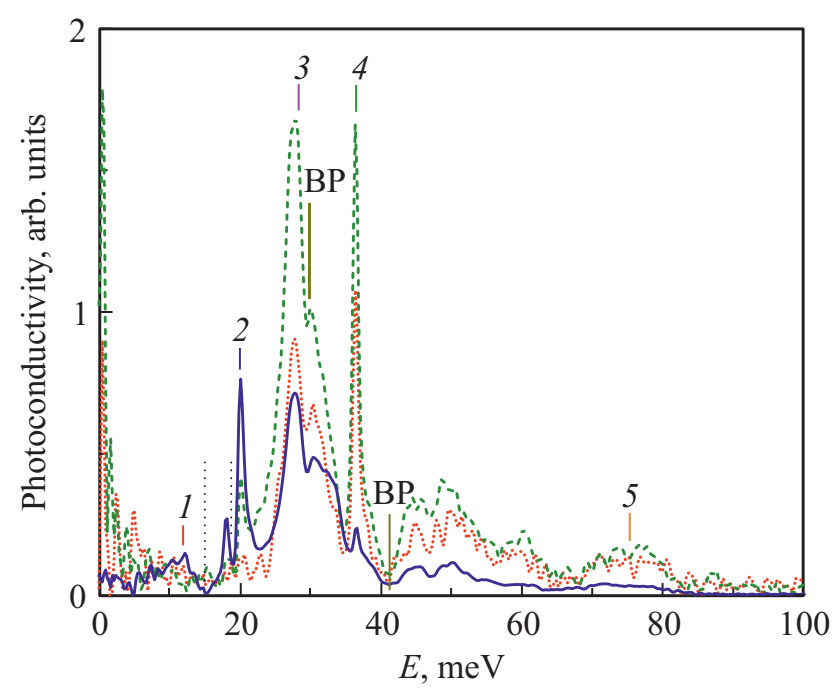

Рис. 3. Спектры примесной фотопроводимости в образце $B$ при различных температурах: сплошная линия - $5 \mathrm{~K}$, штриховая линия $-10 \mathrm{~K}$, пунктирная линия $-11.5 \mathrm{~K}$. Цифрами отмечены наблюдаемые особенности. Точечными вертикальными линиями отмечены области фононного поглощения. ВР области поглощения используемого черного полиэтилена.

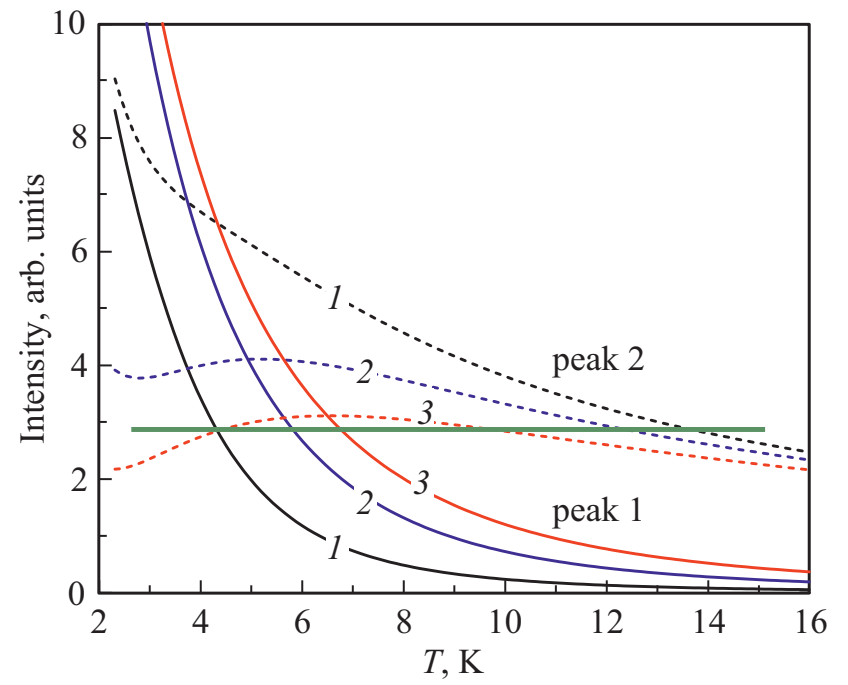

Рис. 4. Рассчитанное соотношение интенсивностей линий 1 (сплошные) и 2 (пунктирные) в зависимости от температуры при различных концентрациях акцепторов $\left(\mathrm{cm}^{-3}\right): 1-4 \cdot 10^{14}$, $2-10^{15}, 3-2 \cdot 10^{15}$. Горизонтальная линия показывает предполагаемый уровень шума.

последнем случае это приводит к кажущемуся появлению пика на 49 мэВ (рис. 2,3), однако это лишь часть полосы особенности 3 .

Увеличение температуры приводит к изменению интенсивностей наблюдаемых особенностей. В образце $A$ рост температуры приводит к постепенному уменьшению всех пиков (рис. 2). В образце $B$ динамика пиков иная: пики 1 и 2 уменьшаются с ростом температуры, в то время как интенсивность пиков 3,4,5 сначала увеличивается, а затем уменьшается. В конечном итоге при достаточно большой температуре ( 12-15K, в зависимости от образца) все особенности исчезают.

Особый интерес представляет эволюция особенностей 1 и 2 , связываемых с вакансиями ртути. С ростом температуры интенсивность этих пиков уменьшается изза температурного гашения и изменения доли состояний многозарядных акцепторов. При некоторой температуре особенность перестает быть различимой в спектрах на фоне шумов. В исследуемых образцах температуры „исчезновения“ особенностей различны: в образце $A$ пик исчезает при $T_{1}^{A} \approx 7 \mathrm{~K}$, а пик 2 при $T_{2}^{A} \approx 10 \mathrm{~K}$ (рис. 2). В образце $B T_{1}^{B} \approx 6 \mathrm{~K}, T_{2}^{B} \approx 12 \mathrm{~K}$ (рис. 3). Таким образом, в образце $B$ температуры „исчезновения“ особенностей отличаются друг от друга сильнее, чем в образце $A$.

Выполненный расчет интенсивности линий, соответствующих $A^{0}$ - и $A^{-1}$-центрам, при различных температурах (рис. 4) показывает, что в зависимости от концентрации акцепторов динамика линий будет различной. При небольшой концентрации акцепторов интенсивность линии 1 , соответствующая ионизации $A^{0}$-центра, будет быстро уменьшаться с ростом температуры, а 
сама ее интенсивность будет значительно меньше интенсивности линии 2, начиная с гелиевых температур (см. кривые 1 на рис. 4).

При большой концентрации акцепторов картина качественно меняется: особенность 1 будет больше особенности 2 при низких температурах $(T<4.5 \mathrm{~K})$, при этом ее интенсивность будет оставаться заметной и при более высоких температурах (см. кривые 3 на рис. 4).

Данные о температуре „исчезновения“ особенностей 1 и 2 в спектрах ФП и расчет интенсивности линий, соответствующих $A^{0}$ - и $A^{-1}$-центрам, позволяют оценить концентрацию акцепторов в наших структурах. Как уже указывалось выше, „исчезновение“ линии - это ее неразличимость на фоне шумов. В наших экспериментах условия измерений (смещение на образце, интенсивность падающего излучения, число усреднений спектров и т.п.) выбирались таким образом, чтобы соотношение сигнал/шум было примерно одинаковым при низкой температуре для разных образцов. Поэтому при анализе расчетных кривых (рис. 4) можно выбирать некоторый уровень шума, по уровню которого определять расчетные температуры „исчезновения“ пиков.

При выборе уровня шума в 2.9 усл.ед. (рис. 4) мы получаем температуры „исчезновения“: $T_{1}=4.3 \mathrm{~K}, T_{2}=13.6 \mathrm{~K}$ при концентрации акцепторов $N_{a}=4 \cdot 10^{14} \mathrm{~cm}^{-3} ; \quad T_{1}=5.8 \mathrm{~K}, \quad T_{2}=12.3 \mathrm{~K} \quad$ при $N_{a}=10^{15} \mathrm{~cm}^{-3} \quad$ и $T_{1}=6.7 \mathrm{~K}, \quad T_{2}=9.5 \mathrm{~K}$ при $N_{a}=$ $=2 \cdot 10^{15} \mathrm{~cm}^{-3}$. Температуры „исчезновения“ $T_{1}$ и $T_{2}$ в последнем случае оказываются очень близки к температурам $T_{1}^{A} \approx 7 \mathrm{~K}$ и $T_{2}^{A} \approx 10 \mathrm{~K}$ для образца $A$. Второй случай соответствует температурам $T_{1}^{B} \approx 6 \mathrm{~K}$, $T_{2}^{B} \approx 12 \mathrm{~K}$ для образца $B$. Таким образом, при выборе единого уровня шума для обоих образцов мы получили согласие между температурами „исчезновения“ обоих пиков 1 и 2. Это позволяет оценить концентрацию акцепторов в образце $A$ как $N_{a}^{A}=2 \cdot 10^{15} \mathrm{~cm}^{-3}$, а в образце $B$ как $N_{a}^{B}=10^{15} \mathrm{~cm}^{-3}$.

\section{5. Заключение}

В работе выполнены исследования спектров фотопроводимости в твердых растворах $\mathrm{Cd}_{x} \mathrm{Hg}_{1-x}$ Tе с долей кадмия $x=0.22-0.23$ в диапазоне температур $T=5-50 \mathrm{~K}$. В спектрах наблюдаются особенности, связанные с межзонным поглощением и переходами с участием примесно-дефектных центров. С ростом температуры красная граница межзонного поглощения сдвигается в сторону больших энергий, положения примесных особенностей не меняются, однако их интенсивность уменьшается. Сдвиг красной границы связан с увеличением ширины запрещенной зоны в КРТ. Уменьшение интенсивности примесных особенностей связано с уменьшением доли неионизованных акцепторных центров, участвующих в формировании сигнала фотопроводимости. В конечном итоге повышение температуры приводит к „исчезновению“ примесных особенностей. Исходя из температур „исчезновения“ особенностей, связанных с вакансиями ртути, была оценена концентрация этих вакансий в рамках простой модели, основанной на уравнении электронейтральности.

\section{Финансирование работы}

Работа выполнена при финансовой поддержке РФФИ (грант № 17-02-00898).

\section{Конфликт интересов}

Авторы заявляют, что у них нет конфликта интересов.

\section{Список литературы}

[1] W. Scott, E.L. Stelzer, R.J. Hager. J. Appl. Phys., 47, 1408 (1976).

[2] D.L. Polla, R.L. Aggarwal, J.A. Mroczkowski, J.F. Shanley, M.B. Reine. Appl. Phys. Lett., 40, 338 (1982).

[3] D.L. Polla, R.L. Aggarwal, D.A. Nelson, J.F. Shanley, M.B. Reine. Appl. Phys. Lett., 43, 941 (1983).

[4] D.L. Polla, R.L. Aggarwal. Appl. Phys. Lett., 44, 775 (1984).

[5] V.V. Rumyantsev, D.V. Kozlov, S.V. Morozov, M.A. Fadeev, A.M. Kadykov, F. Teppe, V.S. Varavin, M.V. Yakyshev, N.N. Mikhailov, S.A. Dvoretskii, V.I. Gavrilenko. Semicond. Sci. Technol., 32, 095007 (2017).

[6] Д.В. Козлов, В.В. Румянцев, С.В. Морозов, А.М. Кадыков, М.А. Фадеев, М.С. Жолудев, В.С. Варавин, Н.Н. Михайлов, С.А. Дворецкий, В.И. Гавриленко, F. Терре. ЖЭТФ, 154, 1226 (2018).

[7] V.S. Varavin, V.V. Vasiliev, S.A. Dvoretsky, N.N. Mikhailov, V.N. Ovsyuk, Yu.G. Sidorov, A.O. Syslyakov, M.V. Yakushev, A.L. Aseev. Proc. SPIE 5136, Solid State Crystals (2002): Crystalline Mater. Optoelectron. (2003).

[8] M.V. Yakushev, M.V. Yakushev, D.V. Brunev, V.S. Varavin, V.V. Vasilyev, S.A. Dvoretskii, I.V. Marchshin, A.V. Predein, I.V. Sabinina, Yu.G. Sidorov, A.V. Sorochkin. Semiconductors, 45, 385 (2011).

[9] В.С. Варавин, Г.Ю. Сидоров Ю.Г. Сидоров. ЖФХ, 84, 1605 (2010).

[10] П.А. Бахтин, С.А. Дворецкий, В.С. Варавин, А.П. Коробкин, Н.Н. Михайлов, И.В. Сабинина, Ю.Г. Сидоров. ФТП, 38, 1207 (2004).

[11] V. Goldman, H. Drew, M. Shayegan D. Nelson. Phys. Rev. Lett., 56, 968 (1986).

[12] P. Capper, J. Garland, S. Kasap, A. Willoughby. Mercury Cadmium Telluride-Growth, Properties and Applications (Wiley, Chichester, 2011).

[13] J.P. Laurenti, J. Camassel, A. Bouhemadou. J. Appl. Phys., 67, 6454 (1990).

[14] M.H. Brodsky, G. Lucovsky. Phys. Rev. Lett., 21, 990 (1968).

[15] D.N. Talwar, M. Vandevyver. J. Appl. Phys., 56, 1601 (1984).

Редактор Г.А. Оганесян 


\section{Evolution of impurity photoconductivity in epitaxial CdHgTe films with temperature}

T.A. Uaman Svetikova ${ }^{1}$, A.V. Ikonnikov' ${ }^{1}$, V.V. Rumyantsev' ${ }^{2}$, D.V. Kozlov ${ }^{2}$, V.I. Chernichkin ${ }^{1}$, A.V. Galeeva ${ }^{1}$, V.S. Varavin ${ }^{3}$, N.N. Mikhailov ${ }^{3}$, S.A. Dvoretskii ${ }^{3}$, S.V. Morozov' ${ }^{2}$, V.I. Gavrilenko ${ }^{2}$

${ }^{1}$ Lomonosov Moscow State University,

119991 Moscow, Russia

${ }^{2}$ Institute for Physics of Microstructures, 603950 Nizhny Novgorod, GSP-105, Russia

${ }^{3}$ Rzhanov Institute of Semiconductor Physics, Siberian Branch of Russian Academy of Sciences, 630090 Novosibirsk, Russia

Abstract The photoconductivity spectra in epitaxial $\mathrm{CdHgTe}$ films at various temperatures were studied by Fourier-transform spectroscopy. Spectral features associated both with interband absorption and with the ionization of impurity/defect states were observed. Their evolution with temperature were traced. The „vanishing“ temperatures of impurity features were determined, which made it possible, using the electroneutrality equation, to estimate the concentration of acceptors in the structures under study. 